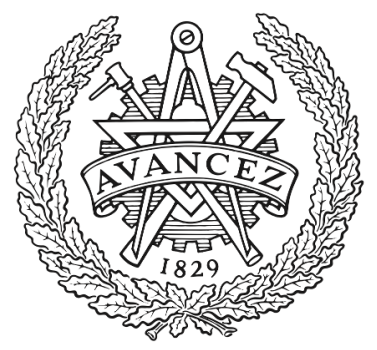

CHALMERS

UNIVERSITY OF TECHNOLOGY

\title{
Generalized robust gain-scheduled PID controller design for affine LPV systems with polytopic uncertainty
}

Downloaded from: https://research.chalmers.se, 2023-04-26 01:36 UTC

Citation for the original published paper (version of record):

Veselý, V., Ilka, A. (2017). Generalized robust gain-scheduled PID controller design for affine LPV systems with polytopic

uncertainty. Systems and Control Letters, 105: 6-13. http://dx.doi.org/10.1016/j.sysconle.2017.04.005

N.B. When citing this work, cite the original published paper. 


\title{
Generalized robust gain-scheduled PID controller design for affine LPV systems with polytopic uncertainty
}

\author{
Vojtech Veselý \\ Institute of Robotics and Cybernetics, Faculty of Electrical Engineering and Information Technology, \\ Slovak University of Technology in Bratislava, Ilkovičova 3, 81219 Bratislava, Slovak Republic \\ Adrian Ilka \\ Department of Signals and Systems, Chalmers University of Technology, Göteborg SE-412 96, Sweden
}

\begin{abstract}
In the paper a generalized guaranteed cost output-feedback robust gain-scheduled PID controller synthesis is presented for affine linear parameter-varying systems under polytopic model uncertainty. The controller synthesis is generalized in a sense that it covers robust, robust gain-scheduled, and robust switched (with arbitrary switching algorithm) PID controller design. The proposed centralized/decentralized controller method is based on Bellman-Lyapunov equation, guaranteed cost, and parameter-dependent quadratic stability. The proposed sufficient robust stability and performance conditions are derived in the form of bilinear matrix inequalities (BMI) which can efficiently be solved or further linearized. As the main result, the suggested performance and stability conditions without any restriction on the controller structure are convex functions of the scheduling and uncertainty parameters. Hence, there is no need for applying multi-convexity or other relaxation techniques and consequently the proposed solution delivers a less conservative design method. The viability of the novel design technique is demonstrated and evaluated through numerical examples.
\end{abstract}

Keywords: Robust controller, Gain-scheduled controller, Switched controller, Parameter-dependent Lyapunov function, Bellman-Lyapunov equation.

\section{Introduction}

Nowadays, gain scheduling techniques for nonlinear systems have been actively researched for about a half a century. These theoretical and application-oriented studies have been accelerated in a great extent by the introduction of the notion of linear parameter-varying (LPV) systems [1]. Basically, there are two main (not independent) research directions for testing and synthetizing performance and stability of LPV systems: Lyapunov theory and

\footnotetext{
Ilka)

Email addresses: vojtech.vesely@stuba.sk (Vojtech Veselý), adrian.ilka@chalmers.se (Adrian 
small-gain theorem. Altough, lot of the LPV design methods are derived by using integral quadratic constraints (IQCs). IQCs allow a dissipation inequality-based interpretation, in which the small-gain condition, the IQCs and the quadratic stability can be integrated in a uniform way (See e.g. [2, 3]). However, the approaches based on IQCs highly depend on the structure of the applied multipliers, therefore may be numerically expensive respectively conservative $[4,5,6]$. According to [6], the standard IQC, in light of conservativeness, is equivalent with the quadratic stability. In addition, authors in [7] stated that their frequencydomain gain-scheduled controller design approach based on the small-gain theorem is likewise on the level of quadratic stability. Nonetheless, these approaches have their own benefits highlighted by a significant amount of publications [8, 9, 10, 11]. For a more comprehensive survey of the field, readers are also referred to survey papers $[12,13,14]$ and references therein.

This paper contributes to controller design techniques using the Lyapunov theory in the affine LPV framework. Within the mentioned framework, convexification in the scheduling and uncertain parameter dependency of the closed-loop conditions ensures obtaining the controller design as an optimization problem subject to some finite number of linear and/or bilinear matrix inequality (LMI/BMI) constraints. Convexity in the scheduling and uncertain parameters can be reached by enforcing the Lyapunov matrix to be constant (i.e. by forcing quadratic stability), e.g. $[15,16]$. In general, the quadratic stability for affine LPV systems makes the controller design quite conservative [12]. However, the conservatism can be relaxed using different types of parameter-varying Lyapunov functions [17, 18]. As pointed out in [17], a multi-convexification technique can balance the conservatism within the affine quadratic stability (AQS) framework. As a result, researchers have started to embed the mentioned multi-convexity requirement in affine LPV framework for gain-scheduled controller, filter, and observer design [17, 19, 20, 21, 22]. Subsequently, in order to further reduce the conservativeness, different techniques to relax the multi-convexity requirement have been deployed [23, 24, 25, 26, 27, 28]. Nevertheless, these relaxations can have significant influence on the performance and can drift the guaranteed cost far away from its optima [29].

Along this line, convexity in the scheduling and uncertain parameters can be ensured also by restricting the closed-loop LPV structure, system or controller to avoid cross term effects of the scheduling parameters. Authors in [30], within the framework of static outputfeedback controller design, ensured convexity under AQS conditions for affine LPV systems by making the static output-feedback controller parameters independent on the scheduled parameters. In [31], the author has restricted the system interconnection with the control input $u(t)$ to be parameter-independent, and hence he annihilated multiplication of the scheduling parameters. The same idea has been applied in [32] and [33] for static outputfeedback controller design with inexact scheduling parameters requiring to solve a set of BMI and LMI conditions, respectively. Similar idea was presented in papers [34] and [35] where some of the dynamic output-feedback LPV controller's matrices were restricted to be parameter-independent in order to obtain convex stability conditions regarding the scheduling parameter.

From this short literature survey follows that there is no general approach in the affine 
LPV framework complemented by the Lyapunov theory, which gives convex dependency on scheduling and uncertain parameters without using the multi-convexity requirement, or major restrictions on the closed-loop system or controller matrices. In addition, only few papers are devoted to LPV-based linear quadratic regulator (LQR) formulation [2, 19, 28, 34, 29]. Triggered by real time applicability, we hereby focus to static, guaranteed cost, fixedorder output-feedback controller design that has not been covered. In this paper, we hence present a new, generalized solution to this specific problem, without any large restrictions in controller or system matrices. The solution is generalized in a sense that it covers robust, robust gain-scheduled, and robust switched (with arbitrary switching sequence) controller design. The control structure is defined as centralized/decentralized fixed-order outputfeedback (similarly to a standard PID) controller. Although, the proposed approach can be used for full/reduced order dynamic output-feedback controller design as well, since it can be reformulated to static output-feedback design [36]. Furthermore, the performance metric is defined in a standard LQR fashion, so the presented approach can be viewed as an extension of the classical infinite-horizon LQR problems [37]. The proposed sufficient robust stability and performance conditions are translated to an optimization problem subject to some LMI and BMI constraints, which can be solved by commercial as well as open source solver tools like PENBMI [38] and PENLAB [39] or can be further linearized. The obtained controller can adapt to changes in the plant dynamics due to the inappropriate scheduling parameters and is robust against any uncertainties in the scheduling measurement of plant modelled in polytopic fashion. Thanks to the polytopic uncertainty representation on the affine LPV system matrices, it is possible to shape the uncertainty polytopes for different working/operation points based on the scheduled parameters. This opens new possibilities in LPV modelling, too.

The paper is organized as follows. Section 2 brings preliminaries and problem formulation. The main generalized robust design procedure is presented in Section 3. Numerical examples illustrate the effectiveness of the proposed approach in Section 4, and concluding remarks close the paper in Section 5.

Our notations are standard. $D \in R^{m \times n}$ denotes the set of real $m \times n$ matrices, $I_{m}$ is an $m \times m$ identity matrix, $P>0(P \geq 0)$ is a real symmetric, positive definite (semidefinite) matrix. Matrices, if not explicitly stated, are assumed to have compatible dimensions.

\section{Preliminaries and problem formulation}

Consider the continuous-time affine linear parameter-varying system with polytopic uncertainty governed by:

$$
\begin{aligned}
& \dot{x}=A(\theta, \xi) x+B(\theta, \xi) u, \\
& y=C x,
\end{aligned}
$$

where $x \in \mathbb{R}^{n}, u \in \mathbb{R}^{m}$ and $y \in \mathbb{R}^{l}$ denote the state, control input and controlled output, respectively. For ease of notation throughout the paper we drop dependency on time. The linear parameter-varying system matrices are assumed to depend on the scheduling vector 
parameter $\theta$ as follows:

$$
A(\theta, \xi)=A_{0}(\xi)+\sum_{j=1}^{p} A_{j}(\xi) \theta_{j} \in \mathbb{R}^{n \times n}, B(\theta, \xi)=B_{0}(\xi)+\sum_{j=1}^{p} B_{j}(\xi) \theta_{j} \in \mathbb{R}^{n \times m} .
$$

Matrices $A_{j}(\xi), B_{j}(\xi), j=0,1,2, \ldots, p$ belong to the convex set, a polytope with $N$ vertices that can be formally defined as:

$$
\begin{aligned}
& \Psi:=\left\{A_{j}(\xi) \in \mathbb{R}^{n \times n}, B_{j}(\xi) \in \mathbb{R}^{n \times m}: A_{j}(\xi), B_{j}(\xi)\right. \\
= & \left.\sum_{i=1}^{N}\left(A_{j i}, B_{j i}\right) \xi_{i}, \sum_{i=1}^{N} \xi_{i}=1, \xi_{i} \geq 0, j=0,1,2, \ldots, p\right\},
\end{aligned}
$$

where $p$ is the number of scheduled parameters, $\xi_{i}, i=1,2, \ldots, N$ are constant or timevarying but unknown parameters, respective uncertainties in system matrices $A_{i}(\xi), B_{i}(\xi)$. Furthermore, $A_{j i}, B_{j i}, C$ are constant matrices of corresponding dimensions. $\theta \in \mathbb{R}^{p}$ is a vector of known constant or time-varying real gain-scheduled parameters. We assume that both lower and upper bounds are available for these parameters and their variation rates that is:

- each parameter $\theta_{j}, j=1,2, \ldots, p$ ranges between known extremal values:

$$
\theta_{j} \in \Omega_{\theta}:=\left\{\theta_{j} \in\left\langle\underline{\theta_{j}}, \overline{\theta_{j}}\right\rangle, j=1,2, \ldots, p\right\},
$$

- the rate of variation $\dot{\theta}_{j}$ is well defined at all times and satisfies:

$$
\dot{\theta}_{j} \in \Omega_{t}:=\left\{\dot{\theta}_{j} \in\left\langle\underline{\theta_{j}}, \overline{\theta_{j}}\right\rangle, j=1,2, \ldots, p\right\} .
$$

Note 1. The uncertain system (1) consists of two types of vertices. The first one is due to the gain-scheduled parameter $\theta$ with $T=2^{p}$ vertices, and the second set of vertices are due to the system uncertainties, $N$-vertices.

Note 2. For the gain-scheduled I part of controller design, the plant states of system (1) need to be extended in such a way that the static output feedback control algorithm can provide proportional (P) and integral (I) parts of the designed PID robust controller. Therefore, throughout the paper is assumed that the plant outputs of system (1) allow us to design the PI controller with static output feedback. For more detail see [40].

The following problem is studied in this paper:

Problem 1. Design a robust static output feedback gain-scheduled PID controller with control algorithm:

$$
u=K(\theta) y+K_{d}(\theta) \dot{y}=K(\theta) C x+K_{d}(\theta) C_{d} \dot{x},
$$

where $K(\theta)=K_{0}+\sum_{j=1}^{p} K_{j} \theta_{j}, K_{d}(\theta)=K_{d 0}+\sum_{j=1}^{p} K_{d j} \theta_{j}$ such that the controller (6) ensures closed-loop system robust parameter-dependent quadratic stability and guaranteed cost with respect to performance (7). The robust stability conditions of gain-scheduled PID controller need to be in convex form regarding scheduled and uncertain parameters $\theta$ and $\xi$. 
To assess performance quality, a quadratic cost function is used:

$$
J_{c}=\int_{0}^{\infty} J(x, u, \dot{x}, \theta) d t
$$

where

$$
J(x, u, \dot{x}, \theta)=x^{T} Q(\theta) x+\dot{x}^{T} S(\theta) \dot{x}+u^{T} R u,
$$

furthermore,

$$
Q(\theta)=Q_{0}+\sum_{j=1}^{p} Q_{j} \theta_{j} \in \mathbb{R}^{n \times n}, S(\theta)=S_{0}+\sum_{j=1}^{p} S_{j} \theta_{j} \in \mathbb{R}^{n \times n},
$$

are positive definite (semidefinite) matrices and $R \in \mathbb{R}^{m \times m}$ is a positive definite matrix.

Remark 1. Using the guaranteed cost approach, the controller parameter's tuning is replaced by the tuning of the weighting parameters. This can relevantly reduce the time and complexity of the tuning process, mainly for large-scale multi-input multi-output applications. For more information and tuning approaches the readers are referred to books [37, 41] and references therein. Regarding the parameter-varying weighting matrices and their benefits, more information can be found in papers [29, 42].

\section{Main results}

This section formulates the theoretical approach to the robust, robust gain-scheduled, and robust switched PID controller design for the uncertain affine LPV system (1), which ensures parameter-dependent quadratic stability and guaranteed cost of the closed-loop system, for all uncertain plant parameters $\Pi \in \Omega$, gain-scheduled parameters $\theta \in \Omega_{\theta}$ and $\dot{\theta} \in \Omega_{t}$. The main results are built on the following Lemma:

Lemma 1. (Bellman-Lyapunov equation) [43] Consider the system (1) with control algorithm (6). Control algorithm (6) is the guaranteed cost control law for the closed-loop system if and only if a Lyapunov function $V(x, \theta, \xi)$ exists such that the following condition holds:

$$
\begin{gathered}
B_{e}=\frac{d V(x, \theta, \xi)}{d t}+J(x, u, \dot{x}, \theta)=-\varepsilon x^{T} x, \\
\varepsilon \mapsto 0, \varepsilon \geq 0 .
\end{gathered}
$$

Proof. Suppose that $P(\theta, \xi)>0$, and the Lyapunov function candidate $V(x, \theta, \xi)=x^{T} P(\theta, \xi) x$ is positive definite. From Eq. (9) for $\epsilon \rightarrow 0$ we can obtain

$$
\dot{V}(x, u, \theta, \xi)+J(x, u, \theta, \xi) \leq 0 \rightarrow \dot{V}(x, u, \theta, \xi) \leq-J(x, u, \theta, \xi) \leq 0 .
$$

Integrating both sides from 0 to $\infty$ we can obtain the upper bound on the cost function:

$$
\begin{aligned}
J_{c}=\int_{0}^{\infty} J(x, u, \theta, \xi) d t=\int_{0}^{\infty}\left(x^{T} Q(\theta) x+u^{T} R u+\dot{x}^{T} S(\theta) \dot{x}\right) d t & \leq V(0)-V(\infty) \\
& \leq V(0)=x_{0}^{T} P(\theta(0), \xi(0)) x_{0} .
\end{aligned}
$$

Since, the initial conditions of the scheduled and uncertain parameters are usually not available, the upper bound on the cost function can be rewritten as $J_{c} \leq \max \left(x_{0}^{T} P(\theta, \xi) x_{0}\right)$. 
Note 3. Equation (9) is known as the Bellman-Lyapunov equation, and function $V(x, \theta, \xi)$ which satisfies (9) is the Lyapunov function. For a particular structure of the Lyapunov function $V(x, \theta, \xi)$, the obtained gain-scheduled controller design procedure may reduce from "if and only if" to "if".

The uncertain system (1) with control algorithm (6) conforming Lemma 1 is called robust parameter-dependent quadratically stable with guaranteed cost. The main results of the robust gain-scheduled PID controller design, which ensure robust parameter-dependent quadratic stability and guaranteed cost, are given in the next theorem.

Theorem 1. The uncertain system (1) is robust parameter-dependent quadratically stable with guaranteed cost, if for the given weighting matrices $Q(\theta), S(\theta), R$ and for the given bounds on $\max \left|\dot{\theta}_{j}\right| \leq \rho_{j}$ there exist auxiliary matrices $N_{i}, i=1, \ldots, 6$, controller gain matrices $K_{i}, K_{d i}, i=0,1, \ldots, p$, symmetric matrices $P_{i, j}, i=0,1, \ldots, p, j=1, \ldots, N$ such that $P(\theta, \xi) \in R^{n \times n}$ is positive definite, and the inequality (10) holds at the vertices of the scheduled parameters $\theta$.

$$
\begin{gathered}
H_{0 i}+\sum_{j=1}^{p} H_{i j} \theta_{j} \leq 0, \\
i=1,2, \ldots, N ; \quad \theta \in \Omega_{\theta} ; \quad \dot{\theta} \in \Omega_{t},
\end{gathered}
$$

where $H_{0 i}=\left\{h_{0 i k l}\right\}_{3 \times 3}, H_{i j}=\left\{h_{k l i j}\right\}_{3 \times 3}$, and

$$
\begin{aligned}
& h_{0 i 11}=N_{1}^{T}+N_{1}-N_{4}^{T} K_{d 0}^{T} C_{d}-C_{d}^{T} K_{d 0}^{T} N_{4}+S_{0}, \\
& h_{0 i 12}=-N_{1}^{T} A_{0 i}+N_{2}+P_{0 i}-N_{4}^{T} K_{0} C-C_{d}^{T} K_{d 0}^{T} N_{5}, \\
& h_{0 i 13}=-N_{1}^{T} B_{0 i}+N_{3}+N_{4}^{T}-C_{d} K_{d 0}^{T} N_{6}, \\
& h_{0 i 22}=-N_{2}^{T} A_{0 i}-A_{0 i}^{T} N_{2}+\sum_{j=1}^{p} P_{j i} \rho_{j}-N_{5}^{T} K_{0} C-C^{T} K_{0}^{T} N_{5}+Q_{0}, \\
& h_{0 i 23}=-N_{2}^{T} B_{0 i}-A_{0 i}^{T} N_{3}+N_{5}^{T}-C^{T} K_{0}^{T} N_{6}, \\
& h_{0 i 33}=-N_{3}^{T} B_{0 i}-B_{0 i}^{T} N_{3}+N_{6}^{T}+N_{6}+R, \\
& h_{11 i j}=-N_{4}^{T} K_{d j} C_{d}-C_{d}^{T} K_{d j}^{T} N_{4}+S_{j}, \\
& h_{12 i j}=-N_{1}^{T} A_{i j}+P_{i j}-N_{4}^{T} K_{j} C-C_{d}^{T} K_{d j}^{T} N_{5}, \\
& h_{13 i j}=-N_{1}^{T} B_{i j}-C_{d}^{T} k_{d j}^{T} N_{6}, \\
& h_{22 i j}=-N_{2}^{T} A_{i j}-A_{i j}^{T} N_{2}-N_{5}^{T} K_{j} C-C^{T} K_{j}^{T} N_{5}+Q_{j}, \\
& h_{23 i j}=-N_{2}^{T} B_{i j}-A_{i j}^{T} N_{3}-C^{T} K_{j}^{T} N_{6}, \\
& h_{33 i j}=-N_{3}^{T} B_{i j}-B_{i j}^{T} N_{3} .
\end{aligned}
$$

Proof. Assume that the Lyapunov function is in the form:

$$
V(x, \theta, \xi)=x^{T} P(\theta, \xi) x,
$$

where

$$
\begin{gathered}
P(\theta, \xi)=P_{0}(\xi)+\sum_{j=1}^{p} P_{j}(\xi) \theta_{j}, \\
P_{j}(\xi)=\sum_{i=1}^{N} P_{j i} \xi_{i}, j=0,1,2, \ldots, p .
\end{gathered}
$$


The time derivative of the Lyapunov function (11) is given as follows:

$$
\begin{aligned}
& \frac{d V(.)}{d t}=\dot{x}^{T} P(\theta, \xi) x+x^{T} P(\dot{\theta}, \xi) x+x^{T} P(\theta, \xi) \dot{x}
\end{aligned}
$$

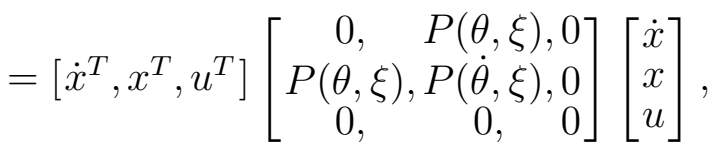

where

$$
P(\dot{\theta}, \xi)=\sum_{j=1}^{p} P_{j}(\xi) \dot{\theta}_{j} \leq \sum_{j=1}^{p} P_{j}(\xi) \rho_{j},
$$

assuming that $P_{j}(\xi) \geq 0$ and $0<\max \left|\dot{\theta}_{j}\right| \leq \rho_{j}$.

To separate matrix $P($.) from the system's matrices $A($.$) and B($.$) , new auxiliary matrices$ $N_{i} \in R^{n \times n}, i=1,2 ; N_{3} \in R^{n \times m} ; N_{i} \in R^{m \times n}, i=4,5 ; N_{6} \in R^{m \times m}$ of corresponding dimensions are introduced in the following form:

$$
\begin{gathered}
2\left(N_{1} \dot{x}+N_{2} x+N_{3} u\right)^{T}(\dot{x}-A(\theta, \xi) x-B(\theta, \xi) u)=0, \\
2\left(N_{4} \dot{x}+N_{5} x+N_{6} u\right)^{T}\left(u-K(\theta) C x-K_{d}(\theta) C_{d} \dot{x}\right)=0 .
\end{gathered}
$$

Summarizing equations (13) with the time derivative of the Lyapunov function (12) one obtains:

$$
\dot{V}(x, \theta, \xi)=z^{T} W(\theta, \xi) z
$$

where $z^{T}=\left[\dot{x}^{T}, x^{T}, u^{T}\right], W(\theta, \xi)=\left\{w_{i j}(\theta, \xi)\right\}_{3 \times 3}$ and

$$
\begin{aligned}
& w_{11}(\theta, \xi)=N_{1}^{T}+N_{1}-N_{4}^{T} K_{d}(\theta) C_{d}-C_{d}^{T} K_{d}(\theta)^{T} N_{4}, \\
& w_{12}(\theta, \xi)=-N_{1}^{T} A(\theta, \xi)+N_{2}+P(\theta, \xi)-N_{4}^{T} K(\theta) C-C_{d}^{T} K_{d}(\theta)^{T} N_{5}, \\
& w_{13}(\theta, \xi)=-N_{1}^{T} B(\theta, \xi)+N_{3}+N_{4}^{T}-C_{d}^{T} K_{d}(\theta)^{T} N_{6}, \\
& w_{22}(\theta, \xi)=-N_{2}^{T} A(\theta, \xi)-A(\theta, \xi)^{T} N_{2}+P(\theta, \xi)-N_{5}^{T} K(\theta) C-C^{T} K(\theta)^{T} N_{5}, \\
& w_{23}(\theta, \xi)=-N_{2}^{T} B(\theta, \xi)-A^{T}(\theta, \xi) N_{3}+N_{5}^{T}-C^{T} K(\theta)^{T} N_{6}, \\
& w_{33}(\theta, \xi)=-N_{3}^{T} B(\theta, \xi)-B(\theta, \xi)^{T} N_{3}+N_{6}^{T}+N_{6} .
\end{aligned}
$$

System (1) with control algorithm (6) is parameter-dependent quadratically stable for all $\theta \in \Omega_{\theta}, \dot{\theta} \in \Omega_{t}$ and uncertainty $\xi$, if matrix $W(\theta, \xi)$ is negative definite (semidefinite). To obtain the guaranteed cost control algorithm, let's substitute the time derivative of the Lyapunov function (14) and performance index (7) to the Bellman-Lyapunov equation (9):

$$
B_{e}=\dot{V}(x, \theta, \xi)+J(x, u, \dot{x}, \theta)=z^{T} H(\theta, \xi) z \leq 0,
$$

where $H(\theta, \xi)=\left\{h_{i j}(\theta, \xi)\right\}_{3 \times 3}$ and

$$
\begin{aligned}
& h_{11}(\theta, \xi)=w_{11}(\theta, \xi)+S(\theta), h_{12}(\theta, \xi)=w_{12}(\theta, \xi), \\
& h_{13}(\theta, \xi)=w_{13}(\theta, \xi), \quad h_{22}(\theta, \xi)=w_{22}(\theta, \xi)+Q(\theta) \text {, } \\
& h_{23}(\theta, \xi)=w_{23}(\theta, \xi), \quad h_{33}(\theta, \xi)=w_{33}(\theta, \xi)+R \text {. }
\end{aligned}
$$


Matrix $H(\theta, \xi)$ is linear with respect to gain-scheduled parameter $\theta$ and uncertainty $\xi$, therefore $H(\theta, \xi)$ can be split into:

$$
H(\theta, \xi)=H_{0}(\xi)+\sum_{j=1}^{p} H_{j}(\xi) \theta_{j}=\sum_{i=1}^{N}\left(H_{0 i}+\sum_{j=1}^{p} H_{i j} \theta_{j}\right) \xi_{i} \leq 0 .
$$

In general, this inequality is an infinite set BMI problem according to scheduled parameter $\theta$ and uncertain parameter $\xi$. However, this inequality (16) is convex with respect to gainscheduled parameter $\theta$ and uncertain parameter $\xi$, therefore (16) holds if and only if it is negative definite (semidefinite) for all $i=1,2, \ldots, N$ ( $\xi$ - vertices) and $j=1,2, \ldots, p$ $(\theta$-vertices).

Remark 2. For the case of $\theta_{j}=0, \rho_{j}=0, j=1,2, \ldots, p$ from (10) one obtains the new procedure to robust PID controller design for the linear time-invariant system with polytopic uncertainty, guaranteed cost, and parameter-dependent quadratic stability. The robust stability condition is in the form:

$$
H_{0 i} \leq 0, \quad i=1,2, \ldots, N \text {. }
$$

Remark 3. Assume that instead of (4) holds

$$
\sum_{j=1}^{p} \theta_{j}=1, \quad \sum_{j=1}^{p} \dot{\theta}_{j}=0, \quad \theta_{j} \in\langle 0,1\rangle .
$$

The gain-scheduled parameter $\theta$ changes to switching variable with arbitrarily switching algorithm. For ideal switching, the rate of $\theta$ change is infinite (positive-negative). In this case one substitute to (10) and $h_{0 i 22}, P_{j i}=0, j=1,2, \ldots, p, i=1,2, \ldots, N$. In the case of non-ideal switching, the rate of $\theta$ changes is finite that is, in (10) the coefficients $\rho_{j} \geq 0$, $j=1,2, \ldots, p$. The number of $\theta$-vertices decreases to $p$. For this case, inequality (10) serves for the design of robust PID switching controller with " $p$ " mode and continuous timeinvariant system. For both cases the designed switched controller ensure guaranteed cost and multi-parameter-dependent quadratic stability to the closed-loop switching system.

Remark 4. For the case of PI controller design matrix $C_{d}=0$ and controller matrices $K_{d i}=0, i=0,1, \ldots, p$. For $P$ or $P D$ controller design the system is simply not extended with the integral part. Furthermore, if the derivative part of the controller includes some filter, the model of this filter can be included in the system model.

Remark 5. The Theorem 1 can be used also for full/reduced order dynamic output-feedback gain-scheduled controller design, since it can be reformulated to static output-feedback design [36].

Because the Theorem 1 is formulated as a feasibility problem, the next corollary completes it with minimization of the LQ cost introduced in (7). 
Corollary 1. For the uncertain affine LPV system (1), an optimal (subotpimal) controller exists in the form (6), if for the given weighting matrices $(Q(\theta), S(\theta)$ and $R$ ) and for the given bounds on $\max \left|\dot{\theta}_{j}\right| \leq \rho_{j}, j=1, \ldots, p$, the following conditions hold:

$$
\min _{F, N, P}(\operatorname{trace}(P(\theta, \xi)))
$$

subject to:

$$
\begin{aligned}
& P(\theta, \xi)>0 \\
& H(\theta, \xi) \leq 0 .
\end{aligned}
$$

Remark 6. Because the initial conditions of the scheduling variables are usually not available, it is recommended to minimize the sum of $\operatorname{trace}(P(\theta, \xi))$ at the corners of the scheduled and uncertain parameters.

\section{Examples}

In order to show the viability of the previous proposed method, the following three examples have been chosen. Numerical solutions have been carried out by PENBMI 2.1 [38] solver under MATLAB 2014b using YALMIP R20150918 [44]. The simulations were done via MATLAB/SIMULINK.

\subsection{Example 1}

Consider the following uncertain (academic) SISO linear parameter-varying continuoustime system in the form of (1) as follows:

$$
A(\theta, \xi)=A_{0}(\xi)+\sum_{j=1}^{p} A_{j}(\xi) \theta_{j}, B(\theta, \xi)=B_{0}(\xi)+\sum_{j=1}^{p} B_{j}(\xi) \theta_{j}
$$

where

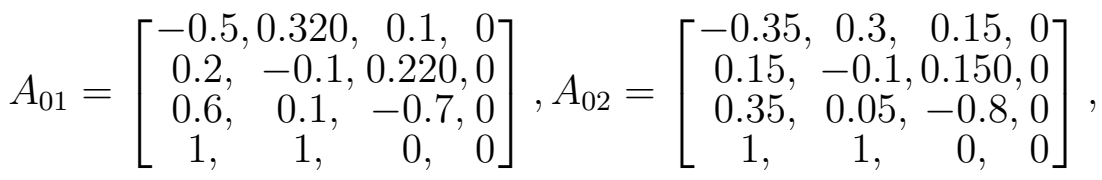

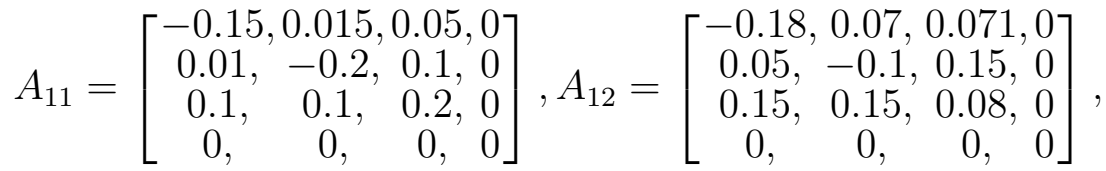

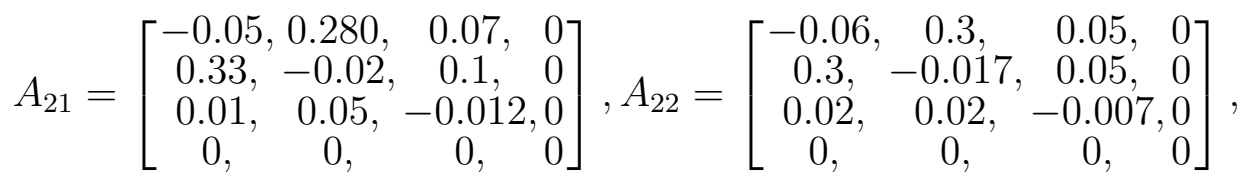

$$
\begin{aligned}
& B_{01}=\left[\begin{array}{l}
0.1 \\
1 \\
0 \\
0
\end{array}\right], B_{02}=\left[\begin{array}{c}
0.15 \\
1.1 \\
0 \\
0
\end{array}\right], B_{11}=\left[\begin{array}{c}
0 \\
0.2 \\
0 \\
0
\end{array}\right], B_{12}=\left[\begin{array}{c}
0.05 \\
0.22 \\
0 \\
0
\end{array}\right], B_{21}=\left[\begin{array}{c}
0.05 \\
0.25 \\
0 \\
0
\end{array}\right], B_{22}=\left[\begin{array}{c}
0.01 \\
0.1 \\
0 \\
0
\end{array}\right] \text {. }
\end{aligned}
$$


Output matrices for PI and derivative (D) part of the controller are as follows:

$$
C=\left[\begin{array}{l}
1,1,0,0 \\
0,0,0,1
\end{array}\right], \quad C_{d}=[1,1,0,0]
$$

The problem is to design the robust PID controller which ensures closed-loop robustness properties, guaranteed cost, and parameter/multi-parameter-dependent quadratic stability for the following cases:

1. Robust switched controller design for the above continuous-time system with two mode and non-ideal arbitrarily switching algorithm. For this case hold $\theta_{1}+\theta_{2}=1, \dot{\theta}_{1}+\dot{\theta}_{2}=0$. The maximal value of the gain-scheduled parameters rate (switching variable rate) is $\max \dot{\theta}_{i}=300 \frac{1}{s}, i=1,2$. Performance parameters of (8) are $Q_{0}=10^{-5} I, Q_{i}=0$, $S_{0}=0, S_{i}=0, i=1,2, R=I$ and $0<P(\theta, \xi)<1000 I$. The obtained switched PID controller (gain-scheduled controller) for the first $\left(\theta_{1}=1, \theta_{2}=0\right)$ and second $\left(\theta_{2}=1, \theta_{1}=0\right)$ mode is given as follows:

$$
R_{\text {switch }}=P_{\text {switch }}+\frac{I_{\text {switch }}}{s}+D_{\text {switch }} s,
$$

where

$$
\begin{aligned}
& P_{\text {switch }}=-2.3257-0.0475 \theta_{1}-0.2613 \theta_{2}, \\
& I_{\text {switch }}=-2.2771-0.0209 \theta_{1}-0.1299 \theta_{2}, \\
& D_{\text {switch }}=+0.0959-0.0420 \theta_{1}-0.0634 \theta_{2} .
\end{aligned}
$$

The simulation results for nominal system with switched robust controller and with randomly generated switching signal are shown on Figs. 1a and 1b, where the dashed blue line is the setpoint $w(t)$, the red line is the system output $y(t)$, and the blue and green lines are the switching/scheduling parameters $\theta_{1}$ and $\theta_{2}$.

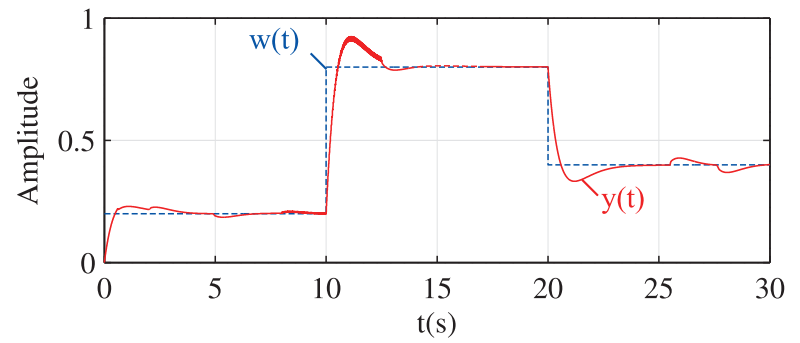

(a) Simulation results $-y(t), w(t)$

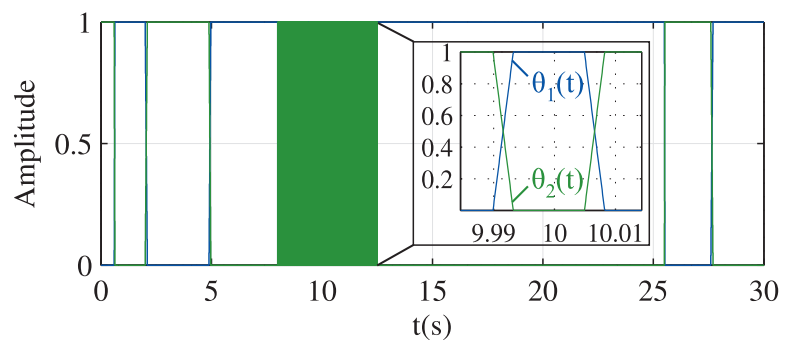

(b) Simulation results $-\theta(t)$

Figure 1: Simulation results for the 1. case

2. Robust gain-scheduled PID Controller design (10) with gain-scheduled parameter rates changes $\dot{\theta}_{1}=5 / \mathrm{s}$ and $\theta_{2}=0$. For the same performance parameters as given above, the obtained gain-scheduled controller is as follows:

$$
R_{G S C}=P_{G S C}+\frac{I_{G S C}}{s}+D_{G S C} s
$$


where

$$
\begin{aligned}
& P_{G S C}=-1.5050+0.0495 \theta_{1}, \\
& I_{G S C}=-0.9728+0.0136 \theta_{1}, \\
& D_{G S C}=-0.1239-0.0412 \theta_{1} .
\end{aligned}
$$

The simulation results for nominal system with robust gain-scheduled controller and with sinuously generated scheduling parameter are shown on Figs. 2a and 2b.

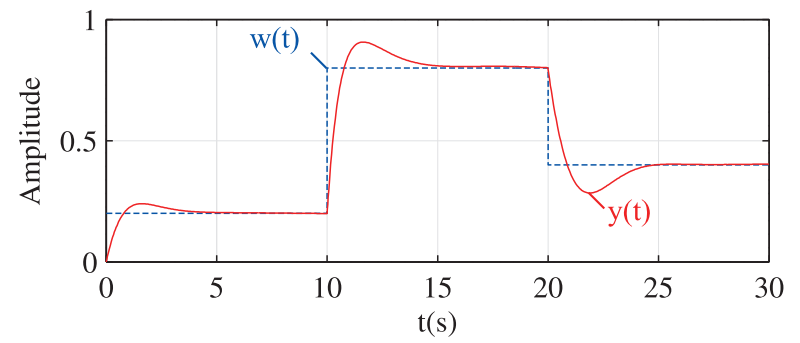

(a) Simulation results $-y(t), w(t)$

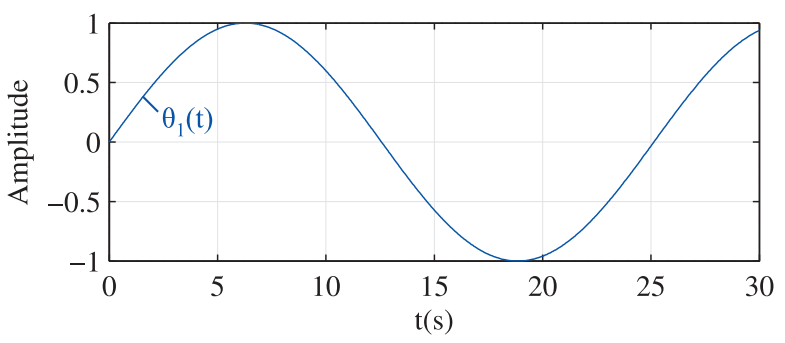

(b) Simulation results $-\theta(t)$

Figure 2: Simulation results for the 2. case

3. Robust PID controller design when $\theta_{1}=\theta_{2}=0$. For the same performance as given in the first example, the obtained robust PID controller which ensures robustness properties of the closed-loop system, guaranteed cost, and parameter-dependent quadratic stability (17) is as follows:

$$
R_{\text {rob }}=-0.9754-\frac{0.5852}{s}-0.0701 s
$$

The simulation result for the nominal system with robust controller and with $\theta_{1}=$ $\theta_{2}=0$ is shown on Fig. 3 .

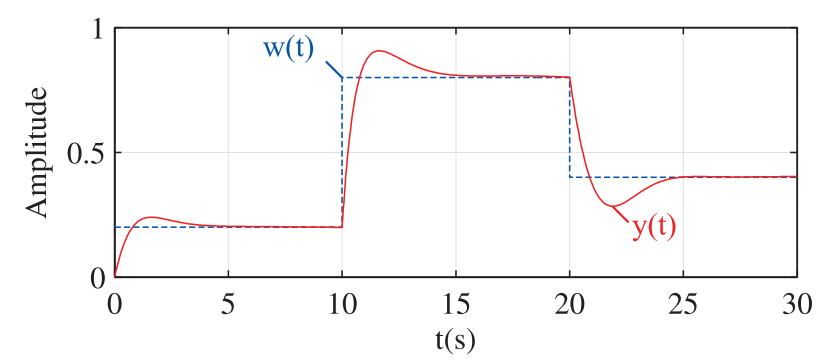

Figure 3: Simulation results $-y(t), w(t)$

\subsection{Example 2}

The second example (levitation problem) is an unstable uncertain dynamic system with one input and one output in the form of (1) as follows: 


$$
\begin{aligned}
& A_{0,1}=\left[\begin{array}{cr}
-4.7500 \times 10^{-5}, 1,0 \\
1, & 0,0 \\
1, & 0,0
\end{array}\right], A_{1,1}=\left[\begin{array}{cc}
1.4250 \times 10^{-4}, 0,0 \\
0, & 0,0 \\
0, & 0,0
\end{array}\right], A_{2,1}=\left[\begin{array}{cr}
-1.0450 \times 10^{-3}, 0,0 \\
0, & 0,0 \\
0, & 0,0
\end{array}\right], \\
& A_{0,2}=\left[\begin{array}{cr}
-5.2500 \times 10^{-5}, 1,0 \\
1, & 0,0 \\
1, & 0,0
\end{array}\right], A_{1,2}=\left[\begin{array}{cc}
1.5750 \times 10^{-4}, 0,0 \\
0, & 0,0 \\
0, & 0,0
\end{array}\right], A_{2,2}=\left[\begin{array}{cr}
-1.1550 \times 10^{-3}, 0,0 \\
0, & 0,0 \\
0, & 0,0
\end{array}\right] \text {, } \\
& B_{0,1}=\left[\begin{array}{c}
0 \\
1.9769 \\
0
\end{array}\right], B_{1,1}=\left[\begin{array}{c}
0 \\
0.2052 \\
0
\end{array}\right], B_{2,1}=\left[\begin{array}{c}
0 \\
-0.15105 \\
0
\end{array}\right] \text {, } \\
& B_{0,2}=\left[\begin{array}{c}
0 \\
2.1850 \\
0
\end{array}\right], B_{1,2}=\left[\begin{array}{c}
0 \\
0.2268 \\
0
\end{array}\right], B_{2,2}=\left[\begin{array}{c}
0 \\
-0.16695 \\
0
\end{array}\right] \\
& C=\left[\begin{array}{l}
1,0,0 \\
0,0,1
\end{array}\right], \quad C_{d}=\left[\begin{array}{l}
1,0,0 \\
0,0,0
\end{array}\right] .
\end{aligned}
$$

We assume that a known vector $\theta(k) \in \Omega$ exists which captures the parametric dependence of the uncertain LPV model as follows:

1. System output: $0.25 M U \longrightarrow \theta_{1}=-1, \theta_{2}=-1$

2. System output: $0.50 M U \longrightarrow \theta_{1}=+1, \theta_{2}=-1$

3. System output: $0.75 M U \longrightarrow \theta_{1}=+1, \theta_{2}=+1$

The problem is to design a robust PID and a robust gain-scheduled PID controller which ensures the closed-loop robustness properties, guaranteed cost, and parameter/multiparameter-dependent quadratic stability.

Using Theorem 1 with weighting matrices $Q=q_{i} I, q_{0}=0.1, q_{1}=q_{2}=0, R=r I, r=1$, $S=s_{i} I, s_{0}=0.1, s_{1}=s_{2}=0,1 \times 10^{-5} \leq P(\theta) \leq 100$ and $\max \theta_{i}=10 \mathrm{~s}^{-1}$ we obtained robust PID controller in the form (6) where

$$
R_{\text {rob }}=-3.4297+\frac{-1.4180}{s}+-2.2529 s,
$$

and robust gain-scheduled PID controller in the form (6) where

$$
R_{G S C}=P_{G S C}+\frac{I_{G S C}}{s}+D_{G S C} s
$$

where

$$
\begin{aligned}
& P_{G S C}=-4.3371+0.3262 \theta_{1}-0.2457 \theta_{2}, \\
& I_{G S C}=-2.1342+0.1633 \theta_{1}-0.1235 \theta_{2}, \\
& D_{G S C}=-3.0851+0.2318 \theta_{1}-0.1748 \theta_{2} .
\end{aligned}
$$

Simulation results are shown on Figs. 4-5, where on Fig. 4a the blue line is the setpoint $w(t)$, the red line is the system output $y_{r o b}(t)$ with the robust PID controller (25) and the green line is the system output $y_{G S}(t)$ with the robust gain-scheduled PID controller (26). Fig. 5a shows the controller output $u_{\text {rob }}(t)$ (magenta) and the calculated scheduled parameters $\theta_{1,2, r o b}(t)$ (red and blue) with the robust PID controller (25). Fig. 5b shows the same for the robust gain-scheduled PID controller (26). Finally Fig. 4b shows the uncertain parameter changes $\xi_{1,2}$. 


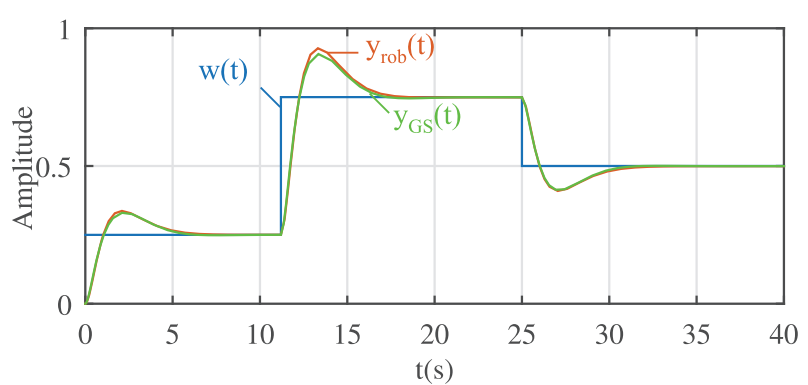

(a) Simulation results $-y_{r o b}(t), y_{G S}(t)$ and $w(t)$

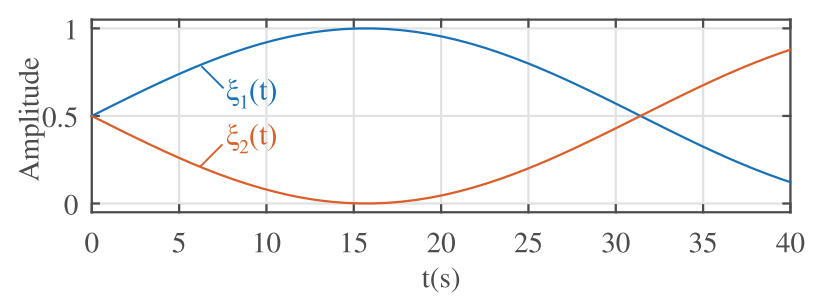

(b) Simulation results $-\xi(t)$

Figure 4: Simulation results - Example 2.

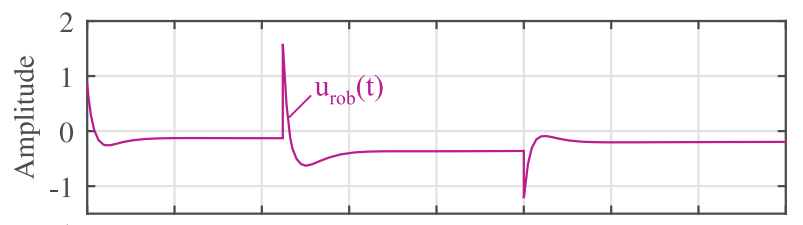

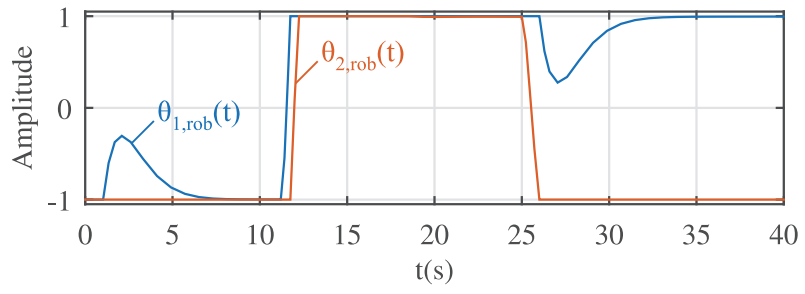

(a) Simulation results $-u_{\text {rob }}(t)$ and $\theta_{r o b}(t)$

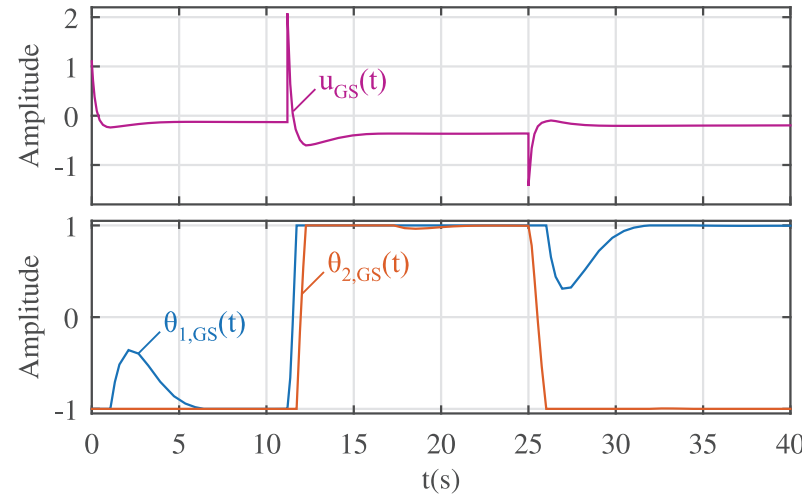

(b) Simulation results $-u_{G S}(t)$ and $\theta_{G S}(t)$

Figure 5: Simulation results - Example 2.

\subsection{Example 3}

Consider 100 randomly generated TITO (two inputs, two outputs), linear continuoustime affine uncertain systems with two uncertainties. If one substitutes the lower and upper bound of uncertainties to the plant model, a polytopic system is obtained. In order to examine the conservativeness of the proposed approach, the affine uncertain systems have been generated to be on the border of feasibility. In the Table 1 one can find the amount of successful solutions for the methods proposed in the paper (by parameter-dependent quadratic stability (PDQS) and quadratic stability (QS)) compared with the classical robust controller method (PDQS) [45]. For decentralized controller design, the following weighting matrices were used: $Q=q_{i} I, q_{0}=0.5, q_{1}=q_{2}=0, R=r I, r=1, S=s_{i} I, s_{0}=0.5$, $s_{1}=s_{2}=0,0.1 \leq P(\theta) \leq 1000$ and $\max \theta_{i}=10 \mathrm{~s}^{-1}$.

Table 1 implies that in case of third or less order TITO plant the classical method is superior and less conservative. However, in case of higher plant orders, the method proposed in this paper gives less conservative results than the classical one. Therefore, the proposed approach contributes to the design tools for robust controllers which guarantees the parameter-dependent quadratic stability, guaranteed cost, and less conservativeness for higher order systems. 
Table 1: Robust PID controller calculation. Results.

\begin{tabular}{cccc}
\hline Amount $\times$ system's order & Prop. met. PDQS & Prop. met. QS & Classical met. PDQS \\
\hline $100 \times 3$ rd & 59 & 59 & 64 \\
$100 \times 4$ th & 63 & 62 & 46 \\
$100 \times 5$ th & 63 & 62 & 49 \\
$100 \times 6$ th & 59 & 55 & 36 \\
\hline
\end{tabular}

\section{Conclusion}

A novel approach to design a robust controller, robust gain-scheduled controller and robust switched controller with arbitrarily switching algorithm is presented. The rate of the switching variable is assumed to be $\dot{\theta}= \pm \infty$ for ideal, and $|\dot{\theta}|<\infty$ for non-ideal switching. The proposed design procedure is based on the Bellman-Lyapunov equation, guaranteed cost, and robust stability conditions using parameter-dependent quadratic stability, or for switched systems the multi-parameter-dependent quadratic stability approach. The obtained feasible robust stability and performance conditions for robust, robust gainscheduled, and robust switched controller design are in the form of BMIs. Despite that the obtained solution is bilinear regarding the variables, the conservativeness of the introduced design procedure is markedly reduced compared with such approaches that use the multiconvexity lemma and/or its relaxations. In our case, the conservativeness is reduced due to the fact that the presented solution is directly convex regarding the scheduled and uncertain parameters. In addition, in the presented solution there is no need for major restrictions in system and/or controller matrices unlike in many references.

Future research will focus on the extension of the established results for systems affected by noises and disturbances, as well as to guarantee the robustness properties and performance of the closed-loop system with hard input constraints. In addition, further emphasis will be put on investigation of the possible LMI solution in order to reduce the remained conservativeness caused by the limitations of currently available BMI solvers.

\section{Acknowledgment}

The work has been supported by the Slovak Scientific Grant Agency, Grant No. 1/0475/16, by Chalmers Area of Advance Transportation, by Vinnova under the FFI project MultiMEC and by Vinnova under FFI project VCloud II.

\section{References}

[1] J. S. Shamma, Control of Linear Parameter Varying Systems with Applications, Springer, 2012, Ch. An overview of LPV systems, pp. 3-26.

[2] F. Wu, Control of Linear Parameter Varying Systems, Ph.D. thesis, University of California, Berkeley (1995). 
[3] P. Seiler, Stability Analysis With Dissipation Inequalities and Integral Quadratic Constraints, IEEE Transactions on Automatic Control 60 (6) (2015) 1704-1709.

[4] A. Packard, Gain scheduling via linear fractional transformations, Systems \& Control Letters 22 (2) (1994) 79-92.

[5] P. Apkarian, P. Gahinet, A convex characterization of gain-scheduled $\mathrm{H} \infty$ controllers, IEEE Transactions on Automatic Control 40 (5) (1995) 853-864.

[6] A. Megretski, A. Rantzer, System analysis via integral quadratic constraints, IEEE Transactions on Automatic Control 42 (6) (1997) 819-830.

[7] J. Osuský, V. Veselý, Robust Gain Scheduling Control Design in Frequency Domain, International Review of Automatic Control (IREACO) 7 (5) (2014) 476-484.

[8] A. Dabiri, B. Kulcsar, H. Koroglu, Distributed LPV State-Feedback Control Under Control Input Saturation, IEEE Transactions on Automatic Control PP (99) (2016) 1-1.

[9] T. Luspay, T. Péni, B. Kulcsár, Control of Linear Parameter Varying Systems with Applications, Springer, 2012, Ch. Constrained freeway traffic control via Linear Parameter Varying Paradigms.

[10] J. Veenman, C. W. Scherer, A synthesis framework for robust gain-scheduling controllers, Automatica 50 (11) (2014) 2799-2812.

[11] S. Wang, H. Pfifer, P. Seiler, Robust synthesis for linear parameter varying systems using integral quadratic constraints, Automatica 68 (2016) 111-118.

[12] G. Wei, Z. Wang, W. Li, L. Ma, A Survey on Gain-Scheduled Control and Filtering for ParameterVarying Systems, Discrete Dynamics in Nature and Society 2014 (2014) 10 pages.

[13] D. J. Leith, W. E. Leithead, Survey of gain-scheduling analysis and design, International Journal of Control 73 (11) (2000) 1001-1025.

[14] W. J. Rugh, J. S. Shamma, Survey Research on gain scheduling, Automatica 36 (10) (2000) 1401-1425.

[15] G. Becker, A. Packard, Robust performance of linear parametrically varying systems using parametrically-dependent linear feedback, Systems \& Control Letters 23 (3) (1994) 205-215.

[16] I. E. Kose, F. Jabbari, W. E. Schmitendorf, A direct characterization of L2-gain controllers for LPV systems, in: 35th IEEE Conference on Decision and Control, Vol. 4, 1996, pp. 3990-3995.

[17] P. Gahinet, P. Apkarian, M. Chilali, Affine parameter-dependent Lyapunov functions and real parametric uncertainty, IEEE Transactions on Automatic Control 41 (3) (1996) 436-442.

[18] F. Wang, V. Balakrishnan, Improved stability analysis and gain-scheduled controller synthesis for parameter-dependent systems, IEEE Transactions on Automatic Control 47 (5) (2002) 720-734.

[19] V. Veselý, A. Ilka, Gain-scheduled PID controller design, Journal of Process Control 23 (8) (2013) $1141-1148$.

[20] Y. Liu, J. Yang, C. Li, Finite-time stability analysis and controller synthesis for switched linear parameter-varying systems, in: 33rd Chinese Control Conference (CCC), 2014, pp. 4360-4365.

[21] M. Sato, Filter design for LPV systems using quadratically parameter-dependent Lyapunov functions, Automatica 42 (11) (2006) 2017-2023.

[22] G. I. Bara, J. Daafouz, F. Kratz, J. Ragot, Parameter-dependent state observer design for affine LPV systems, International Journal of Control 74 (16) (2001) 1601-1611.

[23] P. Apkarian, H. D. Tuan, Parameterized LMIs in Control Theory, SIAM Journal on Control and Optimization 38 (4) (2000) 1241-1264.

[24] H. D. Tuan, P. Apkarian, Relaxations of parameterized LMIs with control applications, International Journal of Robust and Nonlinear Control 9 (2) (1999) 59-84.

[25] H. Ichihara, E. Nobuyama, Difference of multiconvex relaxation of parameterized LMIs: control applications, in: SICE 2003 Annual Conference, Vol. 1, 2003, pp. 150-155.

[26] H. Ichihara, T. Ishii, E. Nobuyama, Stability analysis and control synthesis with D.C. relaxation of parameterized LMIs, in: European Control Conference (ECC), 2003, pp. 2047-2050.

[27] F. D. Adegas, J. Stoustrup, Structured control of affine linear parameter varying systems, in: Proceedings of the 2011 American Control Conference, 2011, pp. 739-744.

[28] V. Veselý, A. Ilka, Design of robust gain-scheduled PI controllers, Journal of the Franklin Institute 352 (4) (2015) 1476-1494. 
[29] A. Ilka, V. Veselý, T. McKelvey, Robust Gain-Scheduled PSD Controller Design from Educational Perspective, in: Preprints of the 11th IFAC Symposium on Advances in Control Education, Bratislava, Slovakia, 2016, pp. 354-359.

[30] N. Aouani, S. Salhi, G. Garcia, M. Ksouri, Static output feedback control for LPV systems under affine uncertainty structure, in: 3rd International Conference on Systems and Control, 2013, pp. 874-879.

[31] M. Sato, Discrete-time Gain-Scheduled Output-Feedback controllers exploiting inexact scheduling parameters, in: IEEE International Symposium on Computer-Aided Control System Design (CACSD), 2011, pp. 1032-1037.

[32] M. Sato, D. Peaucelle, Gain-scheduled output-feedback controllers using inexact scheduling parameters for continuous-time LPV systems, Automatica 49 (4) (2013) 1019-1025.

[33] M. Sato, Gain-scheduled output feedback controllers for discrete-time LPV systems using bounded inexact scheduling parameters, in: 54th IEEE Conference on Decision and Control (CDC), 2015, pp. $730-735$.

[34] V. Veselý, A. Ilka, Unified Robust Gain-Scheduled and Switched Controller Design for Linear Continuous-Time Systems, International Review of Automatic Control (IREACO) 8 (3) (2015) 251-259.

[35] Z. Emedi, A. Karimi, Fixed-structure LPV discrete-time controller design with induced L2-norm and H2 performance, International Journal of Control 89 (3) (2016) 494-505.

[36] D. Petersson, J. Löfberg, LPV H2-controller synthesis using nonlinear programming, in: Proceedings of the 18th IFAC World Congress, 2011.

[37] M. Athans, P. Falb, Optimal control, McGraw-Hill Electrical and Electronik Engineering Series, McGraw-Hill Publishing Company, Ltd, Maidenhead, Berksh, New York, 1966, pp. 879.

[38] D. Henrion, J. Löfberg, M. Kočvara, M. Stingl, Solving polynomial static output feedback problems with PENBMI, in: 44th IEEE Conference on Decision and Control, and European Control Conference. CDC-ECC '05., 2005, pp. 7581-7586.

[39] J. Fiala, M. Kočvara, M. Stingl, Penlab: A matlab solver for nonlinear semidefinite optimization, submitted to Mathematical Programming Computation (October 2013).

[40] V. Veselý, D. Rosinová, Robust PID-PSD Controller Design: BMI Approach, Asian Journal of Control 15 (2) (2013) 469-478.

[41] P. Dorato, C. T. Abdallah, V. Cerone, Linear Quadratic Control: An Introduction, Krieger Publishing Company, 2000.

[42] A. Ilka, V. Veselý, Gain-Scheduled Controller Design: Variable Weighting Approach, Journal of Electrical Engineering 65 (2) (2014) 116-120.

[43] V. M. Kuncevic, M. M. Lycak, Control systems design using Lyapunov function approach, Vol. in Russian, Nauka, Moscow, 1977.

[44] J. Löfberg, YALMIP : A Toolbox for Modeling and Optimization in MATLAB, in: Proceedings of the CACSD Conference, Taipei, Taiwan, 2004.

[45] D. Peaucelle, D. Arzelier, O. Bachelier, J. Bernussou, A new robust D-stability condition for real convex polytopic uncertainty, Systems \& Control Letters 40 (1) (2000) 21-30. 